УДК 611.133.33+611.145.1]-091;614.1

DOI: 10.26435/UC.V0I4(37).537

Р.В. Басий, Ю.В. Довгялло, К.М. Вельма

ГОО ВПО «Донецкий национальный медицинский университет имени М. Горького», Донецк

\title{
ФРАКТАЛЬНЫЙ АНАЛИЗ ГЕОМЕТРИИ ПОВЕРХНОСТНОГО АРТЕРИАЛЬНОГО РУСЛА БОЛЬШИХ ПОЛУШАРИЙ ГОЛОВНОГО МОЗГА
}

\begin{abstract}
Увеличение количества случаев сосудистой патологии требует поиска новых подходов к ее ранней диагностике, в том числе на доклинической стадии. Преобладающая причина смертности во всем мире - ишемический инсульт, поэтому изучение морфофункциональных особенностей артериального русла головного мозга является актуальной задачей современной медицины [1]. С математической точки зрения, поверхностное артериальное русло головного мозга представляет собой фрактальную структуру, равномерность распределения которой на поверхности больших полушарий, ствола мозга и мозжечка может служить морфологической основой оценки адекватности кровоснабжения различных отделов головного мозга [2].

Развитие патологии вне- или внутричерепных артерий может быть причиной упрощения или, наоборот, усложнения организации поверхностного артериального русла, «хаотической» динамики фрактальной геометрии [3, 4]. Перспективные диагностические методы визуализации поверхностных и внутримозговых артерий, несомненно, требуют количественной характеристики этой самой пространственной неоднородности. [5, 6]. Морфометрическим показателем, позволяющим не только визуально, но и количественно, а значит, объективно оценить изменения поверхностной артериальной сети, может служить фрактальный индекс. Однако отсутствие эталона нормы данного маркера для различных отделов поверхностного артериального русла сдерживает использование этого метода для диагностики сосудистой патологии [7]. Рядом с этой проблемой стоит вопрос оценки половых отличий организации поверхностной сосудистой сети и, как следствие, различий в значениях фрактального индекса для лиц мужского и женского пола.
\end{abstract}

\section{ЦЕЛЬРАБОТЫ}

Установить значения показателя фрактального индекса поверхностного артериального русла различных отделов верхнелатеральной поверхности больших полушарий в зависимости от пола.

\section{МАТЕРИАЛ И МЕТОДЫ}

В соответствии с целью и задачами работы был определен фрактальный индекс поверхностного артериального русла верхнелатеральной поверхности головного мозга 24 людей в возрасте 33-65 лет, причина смерти которых не связана с сосудистой патологией, из них 12 мужчин и 12 женщин. Расчет фрактального индекса производился методом box-counting [8].

На первом этапе производилась фотосъемка поверхностной артериальной сети конвекситальной поверхности больших полушарий при помощи зеркального фотоаппарата Nikon 3110 с использованием морфометрического маркера для дальнейших расчетов абсолютных размеров исследуемой области. Обработка и анализ полученных цифровых изображений осуществлялась в компьютерной программе Adobe Photoshop. C помощью экранной пиксельной линейки SPRuler на изображении отмерялись и далее вырезались два участка квадратной формы $4 \times 4$ см (для лобной и теменной долей) и два участка $2 \times 2$ см (для затылочной и височной долей).

На полученное изображение накладывалась морфометрическая сетка, состоящая из четырех квадратов (ячеек) со сторонами размером $1 / 2$ стороны исследуемого участка, и подсчитывалось количество ячеек, в которых находились фрагменты артериального русла (см. рис.).

Далее последовательно накладывались сетки с меньшим размером стороны ячеек - в два, четыре, восемь раз по сравнению с первой. Количество ячеек каждой последующей сетки, следовательно, увеличивалось в четыре раза. Результаты подсчета квадратов сетки, содержащих фрагменты артериального русла, заносились в таблицу и использовались для дальнейшей ста-

(c) Р.В. Басий, Ю.В. Довгялло, К.М. Вельма, 2020 (c) Университетская Клиника, 2020 


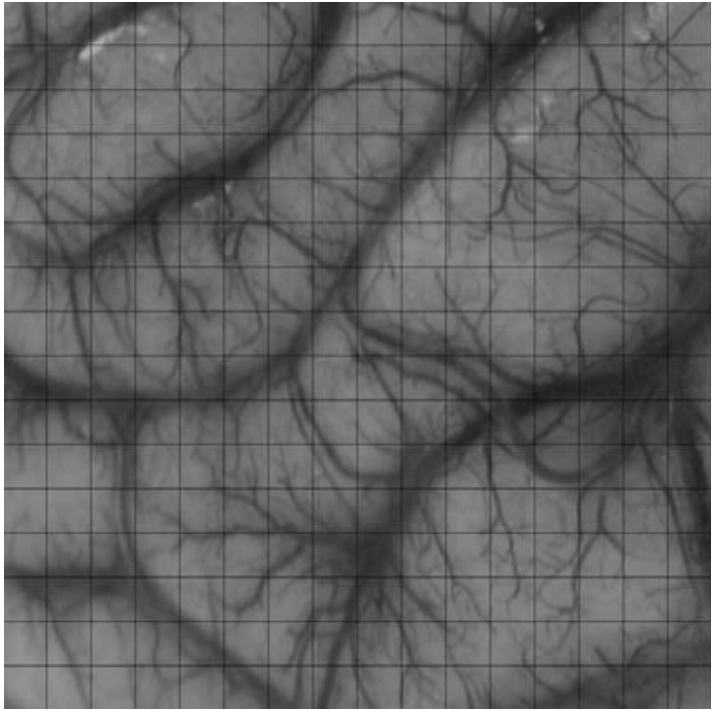

Рис. Один из этапов определения количества ячеек морфометрической сетки, содержащих элементы артериального русла верхнелатеральной поверхности затылочной доли правого полушария.

тистической обработки. Подсчет элементов артериального русла проводился, начиная с третьего этапа, поскольку на первых двух этапах количество ячеек сетки и количество ячеек, содержащих элементы артериального русла, совпадали.

Далее вычислялся натуральный логарифм двух чисел: числа, обратного размеру ячейки, $\ln (1 /$ BoxSize) и числа, соответствующего количеству квадратов сетки, содержащих фрагменты артериальной сети, $-\ln (\mathrm{N})$. На основании полученных данных с помощью компьютерной программы Microsoft Office Excel строился график зависимости $\ln (\mathrm{N})$ от $\ln (1 / \mathrm{BoxSize})$ и рассчитывалось уравнение линейной регрессии. Коэффи- циент перед переменной в уравнении линейной регрессии, определяющий угол наклона графика к оси абсцисс, и представляет собой фрактальный индекс. Статистическая обработка полученных результатов проводилась при помощи лицензионной компьютерной программы MedStat. Выбор того или иного статистического критерия определялся законом распределения частот величин.

\section{РЕЗУЛЬТАТЫ И ОБСУЖДЕНИЕ}

На первом этапе были определены параметры описательной статистики показателей фрактального индекса лобной, теменной, затылочной и височной долей больших полушарий отдельно для лиц мужского и женского пола. Для статистической обработки использовались параметрические критерии, поскольку распределение частот величин изучаемого показателя соответствовало нормальному закону (см. табл.).

Среднее значение фрактального индекса верхнелатеральной поверхности лобной доли больших полушарий составило у мужчин $1,737 \pm 0,03773$, у женщин - $1,8445 \pm 0,03878$, верхнелатеральной поверхности теменной доли у мужчин - $1,715 \pm 0,03602$, у женщин $1,729 \pm 0,02431$, затылочной доли у мужчин $1,827 \pm 0,01395$, у женщин - $1,856 \pm 0,01901$, среднее значение фрактального индекса височной доли оказалось равным $1,786 \pm 0,01752$ у мужчин и $1,818 \pm 0,01987$. При этом размах значений показателя фрактального индекса лобной доли составил 0,42 единицы у мужчин и 0,39 единиц у женщин, теменной доли - 0,42 единицы у мужчин и 0,24 единицы у женщин, затылочной доли $-0,15$ единиц у мужчин и 0,25 единиц у женщин,

Параметры описательной статистики показателя фрактального индекса верхнелатеральной

Таблица. поверхности лобной, теменной и затылочной долей больших полушарий в зависимости от пола $(\mathrm{n}=48)$

\begin{tabular}{lccccccc}
\hline & & Среднее & Минимум & Максимум & $\begin{array}{c}\text { Ошибка } \\
\text { среднего }\end{array}$ & $\begin{array}{c}\text { Левый } \\
\text { ДИ5\%) }\end{array}$ & $\begin{array}{c}\text { Правый } \\
\text { ДИ (95\%) }\end{array}$ \\
\hline \hline \multirow{2}{*}{ Лобная доля } & муж. & 1,737 & 1,5717 & 1,9919 & 0,0377 & 1,654 & 1,821 \\
\cline { 2 - 8 } & жен. & 1,84405 & 1,5046 & 1,8963 & 0,0388 & 1,7432 & 1,794 \\
\hline \multirow{2}{*}{ Теменная доля } & муж. & 1,715 & 1,4719 & 1,8912 & 0,036 & 1,636 & 1,795 \\
\cline { 2 - 8 } & жен. & 1,729 & 1,6083 & 1,8484 & 0,0243 & 1,676 & 1,783 \\
\hline \multirow{2}{*}{$\begin{array}{l}\text { Затылочная } \\
\text { доля }\end{array}$} & муж. & 1,827 & 1,7488 & 1,8946 & 0,01395 & 1,796 & 1,858 \\
\hline \multirow{2}{*}{ Височная доля } & жен. & 1,856 & 1,6681 & 1,9146 & 0,019 & 1,815 & 1,898 \\
\cline { 2 - 8 } & муж. & 1,786 & 1,7131 & 1,825 & 0,0175 & 1,747 & 1,825 \\
\hline
\end{tabular}

Примечания: $\mathrm{n}$ - количество исследованных полушарий головного мозга, 
височной доли - 0,12 единиц у мужчин и женщин.

Однако существенный интерес представлял вопрос наличия или отсутствия достоверных отличий между показателями фрактального индекса верхнелатеральной поверхности у лиц мужского и женского пола. Для определения наличия или отсутствия достоверных отличий использовался параметрический критерий Стьюдента. Оказалось, что значения изучаемого показателя достоверно не отличаются на верхнелатеральной поверхности соответственных долей у мужчин и женщин ( $>>0,05$, где $\mathrm{p}$ - коэффициент достоверности отличий Стьюдента).

Определение фрактального индекса верхнелатеральной поверхности разных долей больших полушарий позволило количественно оценить плотность расположения артериального русла на верхнелатеральной поверхности больших полушарий. Статистические сравнения полученных результатов показали, что значения фрактального индекса не отличаются у лиц мужского и женского пола. Это позволяет говорить о том, что данный морфометрический показатель не зависит от пола, а фрактальный анализ может служить универсальным методом оценки адекватности кровоснабжения больших полушарий головного мозга.
Фрактальный анализ представляет собой еще развивающийся метод оценки свойств и характеристик анатомических структур, который обладает рядом преимуществ, главное из которых - объективность. В контексте использования при исследовании поверхностной артериальной сети больших полушарий этот метод может являться универсальным для лиц обоего пола. Среди перспектив развития данного метода - совершенствование механизма вычисления фрактального индекса и его максимальная автоматизация, разработка эталонных значений фрактального индекса.

\section{З АК ЛЮЧЕ НИЕ}

Современная медицинская наука требует использования новых прогрессивных методов исследования. При этом для получения объективных результатов рационально использовать методы, позволяющие получить количественные характеристики. Также целесообразно применение максимально универсальных методов для простоты и скорости исследования. Одним из таких современных методов определения количественных морфометрических характеристик поверхностного артериального русла больших полушарий является фрактальный анализ.

\section{Р.В. Басий, Ю.В. Довгялло, К.М. Вельма}

ГОО ВПО «Донецкий национальный медицинский университет имени М. Горького», Донецк

\section{ФРАКТАЛЬНЫЙ АНАЛИЗ ГЕОМЕТРИИ ПОВЕРХНОСТНОГО АРТЕРИАЛЬНОГО РУСЛА БОЛЬШИХ ПОЛУШАРИЙ ГОЛОВНОГО МОЗГА}

Рост частоты сосудистой патологии во всем мире заставляет искать новые методы ее ранней диагностики, в том числе на доклинической стадии. Фрактальный анализ является примером объективного метода оценки адекватности кровоснабжения того или иного органа, а значит, может служить маркером морфофункциональных отклонений со стороны артериального русла, в том числе головного мозга. Суть данного метода заключается в определении сложности организации сосудистой сети, меры заполнения пространства исследуемым объектом, в данном случае - элементами артериального русла.

Актуальным для дальнейших исследований является вопрос изучения нормальных показателей фрактального индекса различных отделов поверхностного артериального русла больших полушарий у лиц мужского и женского пола, которые могли бы служить эталоном нормы и использоваться для оценки адекватности кровоснабжения той или иной области конвекситальной поверхности полушарий.

Целью исследования явилось сравнение количественных характеристик поверхностной артериальной сети конвекситальной поверхности больших по- лушарий методом фрактального анализа у лиц мужского и женского пола.

Материал и методы. В соответствии с целью работы было исследовано поверхностное артериальное русло верхнелатеральной поверхности лобной, теменной, затылочной и височной долей 24 людей обоего пола, из них 12 мужчин и 12 женщин. Для расчета фрактального индекса использовался метод box counting. Статистическая обработка полученных peзультатов поводилась при помощи компьютерной программы MedStat.

Результаты. В ходе статистической обработки полученных результатов не было выявлено достоверных отличий между значениями фрактального индекса, характеризующего поверхностное артериальное русло лобной, теменной, затылочной и височной долей у мужчин и женщин, а значит, одно эталонное значение показателя фрактального индекса может использоваться у лиц обоего пола.

Заключение. Современная медицинская наука требует использования новых прогрессивных методов исследования. При этом для получения объективных результатов рационально использовать методы, 
позволяющие получить количественные характеристики. Одним из таких методов является фрактальный анализ.
Ключевые слова: фрактальный анализ, фрактальный индекс, артериальное русло, большие полушария, головной мозг.

\section{R.V. Basii, Yu.V. Dovgyallo, K.M. Vel'ma}

\section{SEI HPE «M. Gorky Donetsk National Medical University», Donetsk}

\section{FRACTAL ANALYSIS OF THE SUPERFICIAL ARTERIAL BED GEOMETRY OF BRAIN HEMISPHERES}

The increasing frequency of vascular pathology throughout the world makes us look for new methods for its early diagnosis, including at the preclinical stage. Fractal analysis is an example of an objective method for assessing the adequacy of blood supply to a particular organ, which means that it can serve as a marker of morphofunctional deviations from the arterial bed, including the brain. An important issue for further research is the study of normal indicators of the fractal index of various departments of the superficial arterial bed of the cerebral hemispheres in males and females, which could serve as a standard and be used to assess the adequacy of blood supply to a particular area of the convexital surface of the hemispheres.

The purpose of the study was to compare the quantitative characteristics of the superficial arterial network of the convexital surface of the cerebral hemispheres by fractal analysis in males and females.

Material and methods. In accordance with the aim of the work, the superficial arterial bed of the upper later- al surface of the frontal, parietal, occipital and temporal lobe of 24 people of both sexes was studied, of which 12 were men and 12 were women. To calculate the fractal index, the box counting method was used. Statistical processing of the results was performed using a licensed computer program MedStat.

Results. During statistical processing of the obtained results, no significant differences were found between the values of the fractal index characterizing the superficial arterial bed of the frontal, parietal, occipital and temporal lobes in men and women, which means that one reference value of the fractal index can be used in people of both sexes.

Conclusion. Modern medical science requires the use of new progressive research methods. Moreover, to obtain objective results, it is rational to use methods that allow to obtain quantitative characteristics. One such method is fractal analysis.

Key words: fractal analysis, fractal index, arterial bed, cerebral hemispheres, brain.

\section{ЛИТЕРАТУРА}

1. Кандыба Д. В. Инсульт. Российский семейный врач. 2016; 20 (3): 5-15.

2. Peyrounette M., Davit Y., Quintard M., Lorthois S. Multiscale modelling of blood flow in cerebral microcirculation: Details at capillary scale control accuracy at the level of the cortex. PLoS One. 2018; 13 (1): e0189474. doi: 10.1371/ journal.pone.0189474

3. De Luca A., Arrigoni F., Romaniello R., Triulzi F. M., Peruzzo D., Bertoldo A. Automatic localization of cerebral cortical malformations using fractal analysis. Phys Med Biol. 2016; 61 (16): 6025-6040. doi: 10.1088/00319155/61/16/6025.

4. Gabriele A. Losa. The living realm depicted by the fractal geometry. Fractal Geometry and Nonlinear Analysis in Medicine and Biology. 2015; 1 (1): 11-15. doi: 10.15761/ FGNAMB.1000104

5. Федер Е. Фракталы. М.: Мир; 1991. 254.

6. Молчатский С. Л. Фрактальная морфология нейронных комплексов ядер гипоталамуса мозга двух представителей млекопитающих. В мире научных открытий. 2014; 10 (58): 146-155.

7. Gao X., Uchiyama Y., Zhou X., Hara T., Asano T., et al. A fast and fully automatic method for cerebrovascular segmentation on time-of-flight (TOF) MRA image. Journal of Digital Imaging. 2010; 24: 609-625.

8. Jiang H., Debuc D. C., Rundek T., Lam B. L., Wright C. B, Shen M., Tao A., Wang J. Automated segmentation and fractal analysis of high-resolution non-invasive capillary perfusion maps of the human retina. Microvasc Res. 2013; 89: $172-175$.

\section{REFERENCES}

1. Kandyba D. V. Insul't. Rossiiskii semeinyi vrach. 2016; 20 (3): 5-15 (in Russian).

2. Peyrounette M., Davit Y., Quintard M., Lorthois S. Multiscale modelling of blood flow in cerebral microcirculation: Details at capillary scale control accuracy at the level of the cortex. PLoS One. 2018; 13 (1): e0189474. doi: 10.1371/ journal.pone.0189474

3. De Luca A., Arrigoni F., Romaniello R., Triulzi F. M., Peruzzo D., Bertoldo A. Automatic localization of cerebral cortical malformations using fractal analysis. Phys Med Biol. 2016; 61 (16): 6025-6040. doi: 10.1088/0031-9155 /61/16/6025.

4. Gabriele A. Losa. The living realm depicted by the fractal geometry. Fractal Geometry and Nonlinear Analysis in Medicine and Biology. 2015; 1 (1): 11-15. doi: 10.15761/ FGNAMB.1000104

5. Feder E. Fraktaly. M.: Mir; 1991. 254.

6. Molchatskii S. L. Fraktal'naya morfologiya neironnykh kompleksov yader gipotalamusa mozga dvukh predstavitelei mlekopitayushchikh. V mire nauchnykh otkrytii. 2014; 10 (58): 146-155 (in Russian).

7. Gao X., Uchiyama Y., Zhou X., Hara T., Asano T., et al. A fast and fully automatic method for cerebrovascular segmentation on time-of-flight (TOF) MRA image. Journal of Digital Imaging. 2010; 24: 609-625.

8. Jiang H., Debuc D. C., Rundek T., Lam B. L., Wright C. B, Shen M., Tao A., Wang J. Automated segmentation and fractal analysis of high-resolution non-invasive capillary perfusion maps of the human retina. Microvasc Res. 2013; 89: $172-175$. 